\title{
Plasma microRNAs levels are different between pulmonary and extrapulmonary ARDS patients: a clinical observational study
}

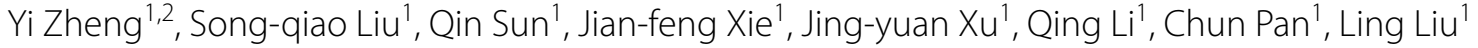 \\ and Ying-zi Huang ${ }^{1 *}$
}

\begin{abstract}
Background: Mesenchymal stem cells (MSC) obviously alleviate the damage of the structure and function of pulmonary vascular endothelial cells (VEC). The therapeutic effects of MSC are significantly different between pulmonary ARDS (ARDSp) and extrapulmonary ARDS (ARDSexp). MicroRNAs (miRNAs), as important media of MSC regulating VEC, are not studied between ARDSp and ARDSexp. We aimed to explore the plasma levels difference of miRNAs that regulate VEC function and are associated with MSC (MSC-VEC-miRNAs) between ARDSp and ARDSexp patients.

Methods: MSC-VEC-miRNAs were obtained through reviewing relevant literatures screened in PubMed database. We enrolled 57 ARDS patients within $24 \mathrm{~h}$ of admission to the ICU and then collected blood samples, extracted plasma supernatant. Patients' clinical data were collected. Then, plasma expression of MSC-VEC-miRNAs was measured by real-time fluorescence quantitative PCR. Simultaneously, plasma endothelial injury markers VCAM-1, vWF and inflammatory factors TNF-a, IL-10 were detected by ELISA method.

Results: Fourteen miRNAs were picked out after screening. A total of 57 ARDS patients were included in this study, among which 43 cases pertained to ARDSp group and 14 cases pertained to ARDSexp group. Plasma miR-221 and miR-27b levels in ARDSexp group exhibited significantly lower than that in ARDSp group (miR-221, 0.22 [0.12-0.49] vs. $0.57[0.22-1.57], P=0.008$, miR-27b, $0.34[0.10-0.46]$ vs. $0.60[0.20-1.46], P=0.025)$. Plasma vWF concentration in ARDSexp group exhibited significantly lower than that in ARDSp group (0.77 [0.29-1.54] vs. 1.80 [0.95-3.51], $P=0.048)$. Significant positive correlation was found between miR-221 and VWF in plasma levels $(r=0.688$,

$P=0.022)$. Plasma miR-26a and miR-27a levels in non-survival group exhibited significantly lower than that in survival group (miR-26a, 0.17 [0.08-0.20] vs. $0.69[0.24-2.33] P=0.018$, miR-27a, 0.23 [0.16-0.58] vs. $1.45[0.38-3.63], P=0.021$ ) in ARDSp patients.
\end{abstract}

Conclusion: Plasma miR-221, miR-27b and VWF levels in ARDSexp group are significantly lower than that in ARDSp group. Plasma miR-26a and miR-27a levels in non-survival group are significantly lower than that in survival group in ARDSp patients.

Keywords: Pulmonary ARDS, Extrapulmonary ARDS, MicroRNA, Vascular endothelial cell

\section{Background}

Acute respiratory distress syndrome (ARDS) is a common critical disease in intensive care unit (ICU). In

\footnotetext{
${ }^{*}$ Correspondence: yz_huang@126.com

${ }^{1}$ Department of Critical Care Medicine, Zhongda Hospital, School of Medicine, Southeast University, No. 87, Dingjiaqiao Road, Gulou District, Nanjing 210009, China

Full list of author information is available at the end of the article
}

recent years, although mechanical ventilation, liquid management, extracorporeal membrane oxygenation and other therapeutic technologies have improved significantly, ARDS is associated with high morbidity and mortality in critically ill patients [1]. Endothelial dysfunction is a key characteristic of ARDS, giving rise to increasing vascular permeability and then pulmonary edema and respiratory failure $[2,3]$. The biological underpinnings 
manipulating the development of endothelial dysfunction in ARDS are incompletely cognized and represent the inevitable course to precision diagnosis and treatment.

Patients with ARDS which is a heterogeneous syndrome have variant etiologies and pathologies and respond differently to therapeutic interventions [4]. One approach to reducing ARDS heterogeneity is to subclassify patients as ARDSp (originating from pulmonary disease) or ARDSexp (originating from extrapulmonary disease) [5]. In the early stages of ARDS, there were significant differences in damage degree of endothelial cells between ARDSp and ARDSexp [6, 7]. When lung morphology was analyzed by computed tomography (CT), ARDSp was characterized by prominent consolidation, while ARDSexp was characterized by prominent groundglass opacification [8]. The two subtypes of ARDS respond differently to therapeutic interventions such as alterations in positive end-expiratory pressure, prone ventilation, and recruitment maneuvers [9-13]. Nevertheless, the underlying mechanism governing this difference needs further research.

Mesenchymal stem cells (MSC), protecting adherens junction (VE-cadherin and $\beta$-catenin), reducing the lung endothelial cell apoptosis, improve pulmonary vascular endothelial cells (VEC) permeability of ARDS [14-18]. However, the therapeutic effects of MSC are significantly different between ARDSp and ARDSexp [19]. This is similar to bone marrow-derived mononuclear cell more effectively improving survival, lung mechanics and histology in ARDSexp than these in ARDSp [20]. The mechanism of difference is not entirely clear.

MicroRNAs (miRNAs), a group of small (19-25 nucleotides) non-coding segments of RNA, regulate gene expression by binding to target mRNA to inhibit their translation. MiRNAs also play an important role in the regulation of gene expression in the pathogenesis of ARDS. Previous studies [21, 22] showed that MSC control activity of pulmonary VEC through regulating microRNAs (miRNAs) levels. Herein, we tentatively defined $M S C$ - $V E C$-miRNAs as a group of miRNAs which are associated with MSC, have regulatory effects on VEC and have previously been studied in ARDS. Then, levels of $M S C-V E C$-miRNAs can be different in patients with ARDSp and ARDSexp.

Yet, so far, no study has tested whether MSC-VECmiRNAs may serve as biomarkers distinguish between ARDSp and ARDSexp. In this study, 14 MSC-VECmiRNAs were filtrated through relevant literatures. Further, we have examined the expression levels of these $M S C$-VEC-miRNAs in plasma collected from patients diagnosed as ARDSp and ARDSexp. Our purpose is to explore the plasma levels difference of $M S C$-VEC-miRNAs between ARDSp and ARDSexp which is probably helpful for the study in pathogenesis and clinical diagnosis of ARDSp and ARDSexp.

\section{Methods \\ Screening of MSC-VEC-miRNA}

Using the combination of keywords and MeSH terms for "endothelial cell" and "microRNA", we searched PubMed for articles that describe associations between the miRNAs and endothelial cell. Each article was reviewed and associated miRNAs ("miRNAs cluster 1") were recorded. Then, we searched each miRNA in "miRNAs cluster 1" individually in conjunction with mesenchymal stromal cell (e.g., "miR-21" and "mesenchymal stromal cell") and reviewed each article to get miRNAs ("miRNAs cluster 2") associated with mesenchymal stromal cell from "miRNAs cluster 1". Using the same method, we obtained $M S C-V E C$-miRNAs, eligible microRNAs that were associated with MSC, has regulatory effects on VEC and has previously been studied in ARDS (Additional file 1: Table S1).

\section{Subject recruitment and sample acquisition}

All new ICU admissions at Zhongda Hospital Affiliated to Southeast University from January 2016 to September 2016 were screened for the presence of ARDS based on acute respiratory distress syndrome: the Berlin Definition [23]. Additional inclusion criteria included 18 years $\leq$ age $\leq 89$ years and admission into the ICU within the previous $24 \mathrm{~h}$. We excluded immunocompromised patients including history of stem cell transplant, immunosuppressive medication using and excluded patients with malignant tumor and pregnant women.

After signing informed consent, subjects had blood drawn via venipuncture or from pre-existing intravascular catheters. Blood samples from enrolled patients were obtained within $24 \mathrm{~h}$ of admission to the ICU. Samples were centrifuged at $1900 \mathrm{~g}$ for $10 \mathrm{~min}$, and the plasma supernatant was extracted and stored in refrigeratory at -80 degrees Celsius.

\section{Patients data collection}

Demographic and clinical data from eligible patients was abstracted from the electronic medical record. Demographic data: gender, age, actual height, actual weight, etc. Patient's condition: main diagnosis, acute physiology and chronic health evaluation (APACHE) II scores, sequential organ failure assessment (SOFA) scores, ARDS etiology. ARDS severity: arterial blood $\mathrm{PO}_{2} / \mathrm{FiO}_{2}$ ratio, Murray lung injury score. The style of oxygen therapy and parameters: noninvasive ventilation, invasive ventilation and ventilator parameters. Clinical outcomes: ICU and hospital length of stay, 28-day mortality, occurrence of shock (defined by clinician), occurrence of acute 
kidney injury [KDIGO Clinical Practice Guideline for Acute Kidney Injury].

\section{RNA isolation}

The frozen plasma was taken out from refrigeratory and incubated at $37{ }^{\circ} \mathrm{C}$ in a water bath until samples are completely thawed. Prolonged incubation should be avoided, which may compromise RNA integrity. RNAs were isolated from plasma samples using miRNeasy serum/ plasma kits (Qiagen). The miRNeasy Serum/Plasma Spike-In Control, a Caenorhabditis elegans miR-39 miRNA mimic, was chosen as the normalized internal control. $3.5 \mu \mathrm{lmiRNeasy} \mathrm{Serum/Plasma} \mathrm{Spike-In} \mathrm{Control}$ $\left(1.6 \times 10^{8}\right.$ copies/ $\mu$ l working solution $)$ was added to the tube containing the lysate before adding chloroform in the RNA extraction process.

\section{Real-time PCR}

After total RNA isolation, quantitative real-time PCR (qRT-PCR) was performed with a miScript System (Qiagen, USA). All procedures were performed according to the instructions provided by the manufacturer. Reverse transcription (RT) was done in a reaction component of $20 \mu \mathrm{l}$, which contained $2 \mu \mathrm{l} \mathrm{miScript} \mathrm{Reverse} \mathrm{Tran-}$ scriptase Mix, $2 \mu \mathrm{l}$ miScript Nucleics Mix, $4 \mu \mathrm{l}$ miScript HiSpec Buffer, a certain volume of template RNA containing $100 \mathrm{ng}$ total RNA and a little RNase-free water increasing reaction volume to $20 \mu \mathrm{l}$. The mixture was incubated $37{ }^{\circ} \mathrm{C}$ for $60 \mathrm{~min}$ and $95^{\circ} \mathrm{C}$ for $5 \mathrm{~min}$. The $20 \mu \mathrm{l}$ RT product was diluted into $100 \mu \mathrm{l}$. Reaction system of quantitative real-time PCR contained $10 \mu \mathrm{l}$ SYBR Green PCR Master Mix, $2 \mu \mathrm{lmiScript} \mathrm{specific} \mathrm{primer,} 2 \mu \mathrm{l} \mathrm{miS-}$ cript universal primer, $2 \mu \mathrm{l}$ cDNA and $4 \mu \mathrm{l}$ RNase-free water. qRT-PCR used an Applied Biosystems StepOne detection system at $95{ }^{\circ} \mathrm{C}$ for $15 \mathrm{~min}$, followed by 40 cycles of $95{ }^{\circ} \mathrm{C}$ for $15 \mathrm{~s}, 55^{\circ} \mathrm{C}$ for $30 \mathrm{~s}, 70{ }^{\circ} \mathrm{C}$ for $30 \mathrm{~s}$. All qRT-PCRs were performed in triplicate, and the raw $\mathrm{Ct}$ (threshold cycle) of each sample was the mean value of three $\mathrm{Ct}$ values. The data were analyzed by the $2^{-\Delta \Delta C T}$ method.

\section{Statistical analysis}

Baseline characteristics and clinical condition indicator of human subjects were compared between ARDSp and ARDSexp. Expression levels of selected miRNAs detected by qRT-PCR were normalized to miR-39 and analyzed using the $2^{-\Delta \Delta C T}$ method. Results for normally distributed continuous variables are presented as mean \pm SD and compared between groups by Student's $t$ tests. Results for non-normally distributed continuous variables are summarized as medians [interquartile ranges] and were compared by Mann-Whitney $U$ tests. Results for categorical variables are presented as sample rate (constituent ratio) and were compared Chi-squared test or Fisher exact test. Logistic regression analysis was carried out to determine the variables that were associated independently with the death of ARDSp patients. We examined whether miR-26a and miR-27a were independent risk factors for the death after adjustment for age and APACHE II score. All tests were two-sided, and $P$ values $<0.05$ were considered statistically significant.

\section{Results}

\section{Screening result of $M S C-V E C$-miRNA}

Fourteen miRNAs were picked out which include miR15a, miR-16, miR-21, miR-24, miR-26a, miR-27a, miR27b, miR-126, miR-146a, miR-150, miR-155, miR-221, miR-223, miR-320. Relevant references were presented with PubMed Unique Identifier in Additional file 2: Table S2. The detail information of these miRNAs is shown in Table 1.

\section{General characteristics of the patients with ARDS}

A total of 101 patients admitted to the ICU of Zhongda Hospital Affiliated to Southeast University from January 2016 to September 2016; diagnosed ARDS were inspected. Ultimately, 44 patients were excluded (30 malignant tumor patients, six patients administered glucocorticoid in the past 6 months, five patients older than 90 years old and three pregnant women). Fifty-seven were included in the study: 43 cases in ARDSp group and 14 cases in ARDSexp group. Age, BMI, APACHE II score, SOFA score, lactic acid, 28-day mortality rate had no statistical difference $(P>0.05)$ between ARDSp and ARDSexp. General data of the 57 ARDS are listed in Table 2.

\section{Comparison of patient's clinical condition indexes between ARDSp and ARDSexp}

Indicators from clinical monitoring and laboratory detection were compared between ARDSp and ARDSexp. Oxygenation index $\left(\mathrm{PO}_{2} / \mathrm{FiO}_{2}\right)$ in ARDSp was lower than that in ARDSexp (145 [119-203] vs. 206 [184-253], $P=0.012$ ). Murray lung injury score in ARDSp was significantly higher than ARDSexp (2.7 [2-3.3] vs. 1.8 [1.3-2.4], $P=0.008) . \mathrm{FiO}_{2}$ and PEEP had no statistical difference between ARDSp and ARDSexp $(P>0.05)$. The proportion of ECMO, CRRT and invasive mechanical ventilation treatment had no statistical difference between ARDSp and ARDSexp $(P>0.05)$. Indexes related to infection and shock had no statistical difference between two groups $(P>0.05)$ (Table 3$)$.

\section{Comparison of plasma MSC-VEC-miRNAs levels between ARDSp and ARDSexp}

Plasma miR-221 and miR-27b levels in ARDSexp group exhibited significantly lower than that in ARDSp group 
Table 1 Summary of candidate MSC-VEC-miRNAs Regulation in vascular endothelial cells

\begin{tabular}{llll}
\hline MiRNAs & Function on angiogenic process & Gene targets & Adjusting direction \\
\hline miR-15a & Inhibits angiogenesis through direct targeting of VEGF and FGF & FGF2, FGFR1, VEGF, VEGFR2 & - \\
miR-16 & Inhibits tumor angiogenesis and EC-mediated angiogenesis in vitro and in vivo & FGF2, FGFR1, VEGF, VEGFR2 & - \\
miR-21 & Induces tumor angiogenesis in vitro & PTEN & + \\
miR-24 & Decreases endothelial cell proliferation & Sp1 & - \\
miR-26a & Prevents endothelial cell apoptosis & TRPC6 & + \\
miR-27a & Promotes EC angiogenesis in vitro & SEMA6A, Spry2, DII4 & + \\
miR-27b & Promotes EC angiogenesis in vitro & SEMA6A, Spry2, DII4 & + \\
miR-126 & Promotes EC angiogenesis in vitro and in vivo & Spred-1, PIK3R2, VCAM-1 & + \\
miR-150 & Restores vascular barrier function & Ang2 & + \\
miR-146a & Promotes senescence of endothelial cells & NOX4 & - \\
miR-155 & Promotes tumor angiogenesis & VHL & + \\
miR-221 & Inhibits EC-mediated angiogenesis in vitro & C-kit, eNOS & - \\
miR-223 & Prevents endothelial cell proliferation & B1 integrin, IGF-1R & - \\
miR-320 & Inhibits diabetic angiogenesis in vitro & IGF-1 & - \\
\hline
\end{tabular}

miR: microRNA, -: Negative regulation, + : Positive adjustment

Table 2 General data comparison between ARDSp and ARDSexp

\begin{tabular}{|c|c|c|c|c|}
\hline Variable & Total $(n=57)$ & $\operatorname{ARDS}_{p}(1)(n=43)$ & $\operatorname{ARDS}_{\exp }(2)(n=14)$ & $P$ value (1) versus (2) \\
\hline \multicolumn{5}{|l|}{ General condition } \\
\hline Age (years) & $59.0 \pm 17.5$ & $56.6 \pm 20.4$ & $63.7 \pm 12.6$ & 0.13 \\
\hline Male n (\%) & $41(71.9 \%)$ & $30(69.8 \%)$ & $11(78.6 \%)$ & 0.52 \\
\hline $\mathrm{BMI}$ & $23.9 \pm 3.6$ & $24.0 \pm 3.8$ & $23.6 \pm 3.0$ & 0.70 \\
\hline APACHE II score & $21.3 \pm 8.4$ & $21.8 \pm 8.5$ & $20.0 \pm 8.4$ & 0.50 \\
\hline SOFA score & $10.4 \pm 4.9$ & $10.4 \pm 4.6$ & $10.3 \pm 5.7$ & 0.93 \\
\hline 28-day mortality & $18(31.6 \%)$ & $14(32.6 \%)$ & $4(22.2 \%)$ & 1.00 \\
\hline \multicolumn{5}{|l|}{ Basic diseases } \\
\hline COPD $n(\%)$ & $1(1.8 \%)$ & $0(0 \%)$ & $1(7.1 \%)$ & 0.25 \\
\hline Hypertension n (\%) & $16(28.1 \%)$ & $13(30.2 \%)$ & $3(21.4 \%)$ & 0.77 \\
\hline CHD n (\%) & $8(14.0 \%)$ & $7(16.3 \%)$ & $1(7.1 \%)$ & 0.68 \\
\hline CVD $n(\%)$ & $8(14.0 \%)$ & $8(18.6 \%)$ & $0(0 \%)$ & 0.19 \\
\hline $\mathrm{DM} n(\%)$ & $12(21.1 \%)$ & $10(23.3 \%)$ & $2(14.3 \%)$ & 0.74 \\
\hline $\mathrm{HBD} n(\%)$ & $7(12.3 \%)$ & $1(2.3 \%)$ & $6(42.9 \%)$ & 0.001 \\
\hline ISD n (\%) & $0(\%)$ & $0(\%)$ & $0(\%)$ & 1.00 \\
\hline \multicolumn{5}{|l|}{ ARDS etiology } \\
\hline $\mathrm{PI} n(\%)$ & $36(63.2 \%)$ & $36(83.7 \%)$ & $0(0 \%)$ & $<0.001$ \\
\hline Inhalation n (\%) & $3(5.3 \%)$ & $3(7.0 \%)$ & $0(0 \%)$ & 0.57 \\
\hline$P C \cap(\%)$ & $4(7.0 \%)$ & $4(9.5 \%)$ & $0(0 \%)$ & 0.515 \\
\hline Sepsis n (\%) & $3(5.3 \%)$ & $0(0 \%)$ & $3(20 \%)$ & 0.016 \\
\hline Pancreatitis n (\%) & $4(7.0 \%)$ & $0(0 \%)$ & $4(26.7 \%)$ & 0.004 \\
\hline EPT $n(\%)$ & $5(8.8 \%)$ & $0(0 \%)$ & $5(33.3 \%)$ & 0.001 \\
\hline Others n (\%) & $2(3.5 \%)$ & $0(0 \%)$ & $2(14.3 \%)$ & 0.057 \\
\hline \multicolumn{5}{|l|}{ Organ dysfunction } \\
\hline Septic shock $n(\%)$ & $22(38.6 \%)$ & 17 (39.5\%) & $5(35.7 \%)$ & 0.23 \\
\hline $\mathrm{AKI} n(\%)$ & $14(24.6 \%)$ & $10(23.3 \%)$ & $4(28.6 \%)$ & 0.97 \\
\hline
\end{tabular}

$B M I$ body mass index, $C O P D$ chronic obstructive pulmonary disease, $A R D S$ acute respiratory distress syndrome, $A K I$ acute kidney injury, $A P A C H E$ acute physiology and chronic health evaluation, SOFA sequential organ failure assessment, $C H D$ coronary heart disease, $C V D$ cerebrovascular disease, $D M$ diabetes mellitus, $H B D$ hepatobiliary diseases, ISD immune system disease, $P I$ pulmonary infection, $P C$ pulmonary contusion, EPT extrapulmonary trauma 
Table 3 Comparison of patient's clinical condition indexes between ARDSp and ARDSexp

\begin{tabular}{|c|c|c|c|c|}
\hline Variable & Total $(n=57)$ & $\operatorname{ARDS}_{p}(1)(n=43)$ & $\operatorname{ARDS}_{\exp }(2)(n=14)$ & $P$ value (1) versus (2) \\
\hline \multicolumn{5}{|l|}{ Lung injury severity } \\
\hline $\mathrm{PH}$ & $7.4[7.35-7.45]$ & $7.41[7.36-7.46]$ & 7.37 [7.32-7.43] & 0.26 \\
\hline $\mathrm{FiO}_{2}$ & $0.5[0.4-0.6]$ & $0.5[0.4-0.6]$ & $0.4[0.4-0.5]$ & 0.06 \\
\hline PEEP(cmH2O) & $8[5-12]$ & $8[5-12]$ & $5[5-12]$ & 0.54 \\
\hline $\mathrm{PO}_{2} / \mathrm{FiO}_{2}(\mathrm{mmHg})$ & 165 [112-211] & 145 [110-203] & 206 [184-253] & 0.012 \\
\hline Murray score & $2.3[1.7-3.1]$ & $2.7[2-3.3]$ & $1.8[1.3-2.4]$ & 0.008 \\
\hline \multicolumn{5}{|l|}{ Infection index } \\
\hline Leukocyte count & 10.4 [6-16.7] & 10.4 [6.4-14.5] & $11.8[5.5-18.3]$ & 0.81 \\
\hline Platelet count & 134 [90-188] & 134 [107-195] & 128 [49-184] & 0.38 \\
\hline CRP & $74[25-128]$ & $74[31-121]$ & 75 [15-141] & 0.87 \\
\hline PCT & $1.3[0.2-12.9]$ & $1.0[0.2-13.1]$ & $1.86[0.7-10.4]$ & 0.60 \\
\hline \multicolumn{5}{|l|}{ Shockindex } \\
\hline $\mathrm{HR}$ & 73 [63-121] & 72 [63-120] & 76 [63-125] & 0.66 \\
\hline $\mathrm{NE}$ & $5[0-27.5]$ & $4[0-20]$ & $5[0-85]$ & 0.21 \\
\hline Lactic acid & $2.1[1-3.1]$ & $2.0[0.9-2.9]$ & $2.2[1.2-5.4]$ & 0.11 \\
\hline \multicolumn{5}{|l|}{ Organ supporting } \\
\hline IMV n (\%) & $40(70.2 \%)$ & $32(74.4 \%)$ & $8(57.1 \%)$ & 0.37 \\
\hline ECMO $n(\%)$ & $12(21.1 \%)$ & $12(27.9 \%)$ & $0(0 \%)$ & 0.065 \\
\hline CRRT n (\%) & $10(17.5 \%)$ & $7(16.3 \%)$ & $3(21.4 \%)$ & 0.97 \\
\hline
\end{tabular}

$\mathrm{PH}$ arterial blood $\mathrm{pH}$ value, $\mathrm{PO}_{2}$ arterial partial pressure of oxygen, $\mathrm{FiO}_{2}$ oxygen concentration, $\mathrm{PEEP}$ positive end expiratory pressure, $\mathrm{Murray}$ score lung injury score used for ARDS patients, CRP C reactive protein, $P C T$ procalcitonin, IMV invasive mechanical ventilation, ECMO extracorporeal membrane oxygenation, $C R R T$ continuous renal replacement therapy, $H R$ heart rate, NE norepinephrine. $P<0.05$ suggests statistical difference

(0.22 [0.12-0.49] vs. 0.57 [0.22-1.57], $P=0.008),(0.34$ [0.10-0.46] vs. $0.60[0.20-1.46], P=0.025)$. Other 12 kinds of plasma miRNAs levels between two groups showed no statistical difference. Plasma levels of $M S C$ $V E C$-miRNAs between ARDSp and ARDSexp are shown in Fig. 1.

\section{Comparison of plasma vWF, VCAM-1, IL10, TNFa concentration between ARDSp and ARDSexp}

Plasma vWF concentration in ARDSexp group exhibited significantly lower than that in ARDSp group (0.77 [0.29$1.54]$ vs. 1.80 [0.95-3.51], $P=0.048$ ). However, VCAM-1, IL10, TNF $\alpha$ concentration between two groups showed no statistical difference. Plasma concentration of VCAM1, IL10, TNF $\alpha$ between ARDSp and ARDSexp is shown in Fig. 2.

\section{The correlation of plasma levels between miR-27b/miR-221} and vWF

As plasma miR-27b/miR-221 and vWF levels were significant different between ARDSp and ARDSexp groups, we analyzed the correlation of plasma levels between miR27b/miR-221 and vWF. We found significant positive correlation between miR-221 and vWF in plasma levels $(r=0.688, P=0.022)$. However, there was no significant correlation between miR-27b and vWF in plasma levels (Fig. 3).

\section{Comparison of plasma patient's clinical illness condition data between survival and non-survival group in ARDSp patients}

APACHE II score, SOFA score, P/F, Murray score, CRP, Lactic acid were used as common indicators to evaluate ARDS patients' clinical illness condition. This study showed that APACHE II score, SOFA score and lactic acid in survival group were significantly lower than that in non-survival group (APACHE II score: $18.7 \pm 7.6$ vs. $28.1 \pm 7.6, P<0.001$; SOFA score: $8.8 \pm 4.1$ vs. $14.0 \pm 3.8$, $P<0.001$; lactic acid: $1.7[0.9-2.2]$ vs. 2.9 [1.2-3.3], $P=0.015)$ in ARDSp patients. $\mathrm{P} / \mathrm{F}$, Murray score and CRP between two groups showed no statistical difference (Table 4).

\section{Comparison of plasma MSC-VEC-miRNAs levels between survival and non-survival group in ARDSp patients}

In our research, extrapulmonary ARDS was caused by sepsis, pancreatitis, extrapulmonary trauma etc. We just analyzed plasma $M S C$-VEC-miRNAs and vWF, VCAM-1, IL10, TNF $\alpha$ levels between 28 days survival and 28 days non-survival group in ARDSp patients in order to reduce the heterogeneity between patients. Plasma miR-26a and miR-27a levels in non-survival group exhibited significantly lower than that in survival group (miR-26a: 0.17 [0.08-0.20] vs. 0.69 [0.24-2.33] $P=0.018$; miR-27a: 0.23 


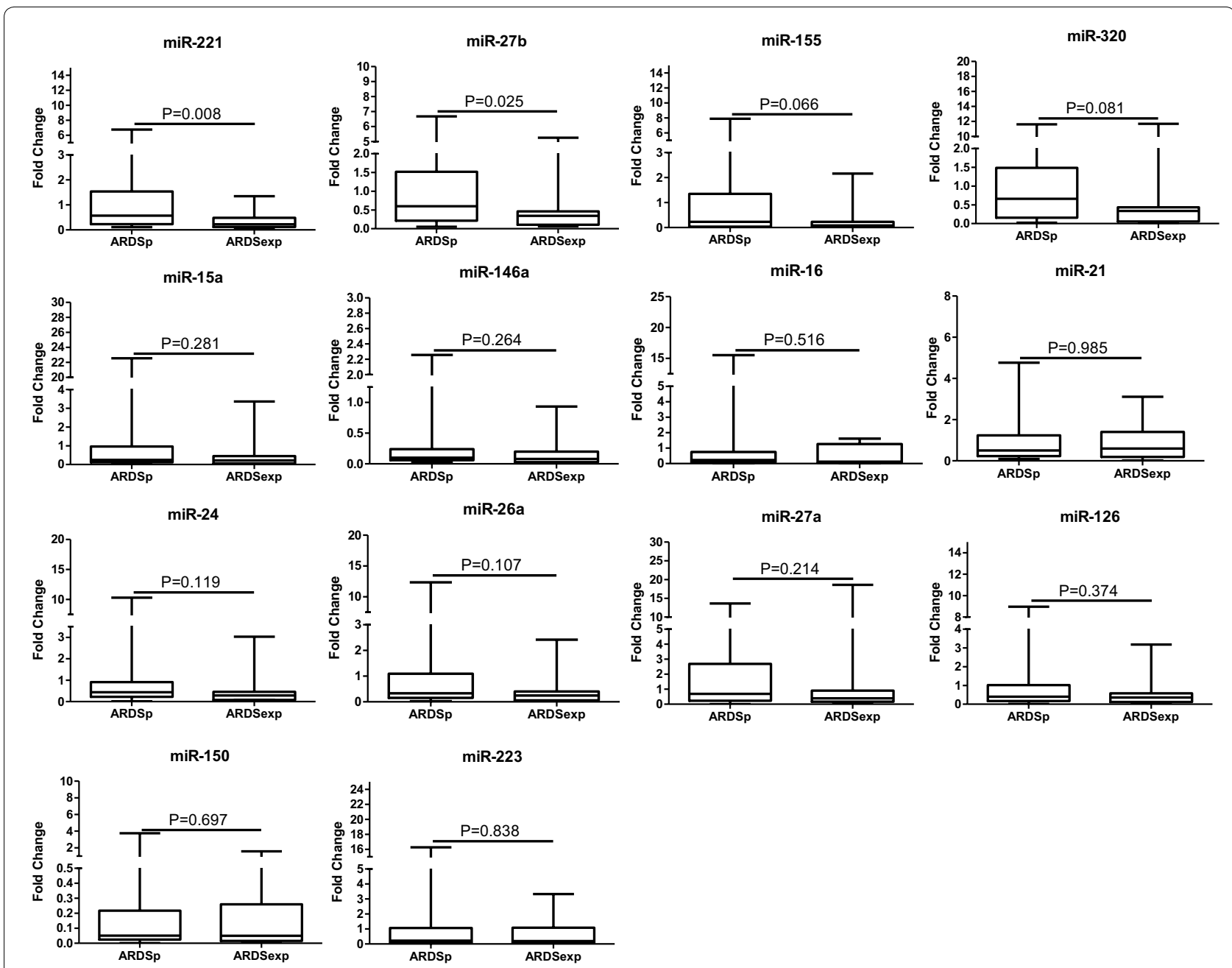

Fig. 1 Comparison of MSC-VEC-miRNAs between ARDSp and ARDSexp. Data presented as a relative fold change between ARDSp and ARDSexp for each miRNA. Box plots are displayed where the horizontal bar represents the median, the box represents the IQR, and the whiskers represent the maximum and minimum values. Comparisons made by Mann-Whitney $U$ test. miRNA microRNA, IQR interquartile range

[0.16-0.58] vs. $1.45[0.38-3.63], P=0.021)$ in ARDSp patients. Other 12 kinds of miRNAs and vWF, VCAM-1, IL10, TNF $\alpha$ levels in plasma between two groups showed no statistical difference (Figs. 4, 5).

\section{The predictive value of miR-26a and miR-27a for prognosis of ARDSp patients}

As APACHE II score, SOFA score, lactic acid, miR$26 \mathrm{a}$ and miR-27a were significantly different between non-survival and survival groups in ARDSp patients, ROC curves were drawn and the area under the curve (AUC) values for APACHE II score, SOFA score, lactic acid, miR-26a and miR-27a were, respectively, 0.808 (95\%CI: $0.673-0.943), \quad 0.828$ (95\%CI: $0.693-0.962)$, 0.782 (95\%CI: 0.564-0.897), 0.787 (95\%CI: 0.650-0.925), 0.782 (95\%CI: 0.650-0.918) (Fig. 6). We also divided the patients into two groups according to median miR-26a or miR-27a value. Survival curve analysis showed that ARDSp patients with lower concentration of miR-26a/ miR-27a had higher mortality (Fig. 7). Tables 5 and 6 show the results of the multivariate logistic regression analysis for the death of ARDSp patients. MiR-26a (OR: 1.483, 95\% CI: $0.999-2.200, P=0.050)$, miR-27a (OR: 1.425, 95\% CI: $1.008-2.015, P=0.045)$ were may independently associated with the death of ARDSp patients.

\section{Discussion}

The results of this study demonstrate that the expression of plasma miR-221, miR-27b and endothelial markers vWF is significantly different between ARDSp and ARDSexp patients. Plasma miR-26a and miR-27a levels showed significantly different between non-survival group and survival group in ARDSp patients. 

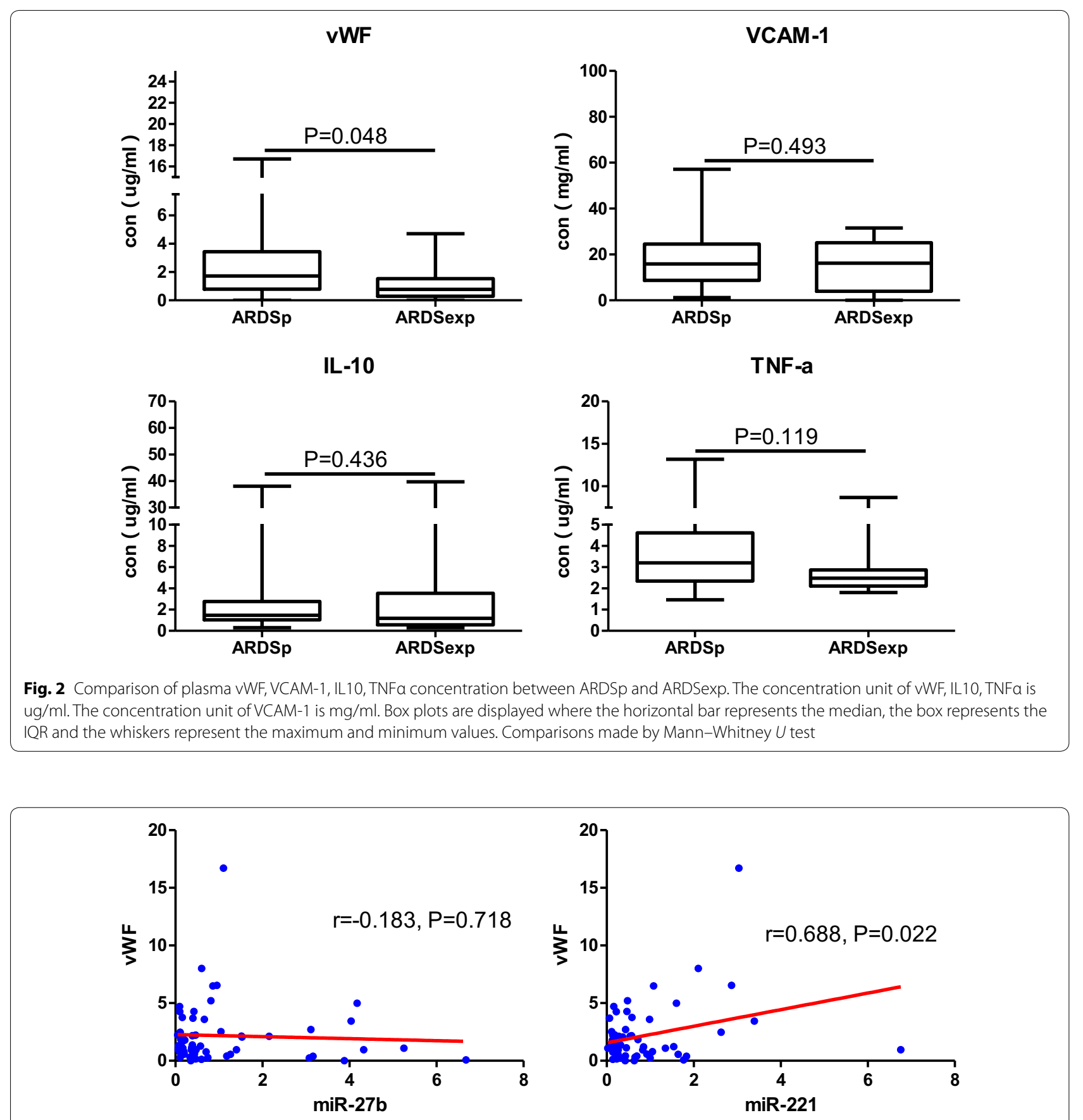

Fig. 3 Correlation between WWF and miR-27b/miR-221

The characteristics of the enrolled patients in this study may impact research results. The ARDSp patients are more serious than the ARDSexp patients in the local lung injury and lung function lesion. The ARDSp patients owned higher Murray lung injury score and lower $\mathrm{PO}_{2} / \mathrm{FiO}_{2}$ than the ARDSexp patients and included all 12 patients received ECMO treatment. But indicators related to the overall illness condition, such as APACHE II scores, SOFA scores, blood lactate levels, doses of norepinephrine and the proportion of complicating sepsis, septic shock, AKI showed no statistical difference between ARDSp and ARDSexp patients. There was no difference in the 28-day mortality between the two groups, probably because the overall illness condition 
Table 4 Comparison of patient's clinical illness condition data in ARDSp patients

\begin{tabular}{lllc}
\hline Variable & Survival $(\boldsymbol{n}=\mathbf{2 9})$ & Non-survival $(\boldsymbol{n}=\mathbf{1 4})$ & $\boldsymbol{P}$ value \\
\hline APACHE II score & $18.7 \pm 7.6$ & $28.1 \pm 7.6$ & $<0.001$ \\
SOFA score & $8.8 \pm 4.1$ & $14.0 \pm 3.8$ & $<0.001$ \\
P/F(mmHg) & $150[113-203]$ & $130[100-195]$ & 0.39 \\
Murray score & $2.6[2.0-3.0]$ & $3.0[2.3-3.7]$ & 0.09 \\
CRP & $73.7[31.0-111]$ & $91.3[33.3-121]$ & 0.76 \\
Lactic acid & $1.7[0.9-2.2]$ & $2.9[1.2-3.3]$ & 0.015 \\
\hline
\end{tabular}

had no difference between the two groups. Therefore, the survival rate depends on overall illness severity or, say, the systematic condition of the whole organ rather than single organ lesions. We should pay attention to primary disease treatment and, meanwhile, systematic organ maintenance to prevent multiple organ dysfunction on critically ill patients.

In our study, pulmonary vascular endothelium lesion in ARDSp patients may be more serious than that in ARDSexp patients which embody in Murray lung injury score and $\mathrm{PO}_{2} / \mathrm{FiO}_{2}$. The result is in agreement with the previous research [24-26]. Previous studies show that miR-27b promotes vascular endothelial cell angiogenesis, yet miR-221 inhibits vascular endothelial cell-mediated angiogenesis. So, we deem ARDSp patients will express higher levels of miR-221 and, conversely, express reduced levels of miR-27b than ARDSexp patients. However, our research shows that plasma miR-221 and miR-27b levels
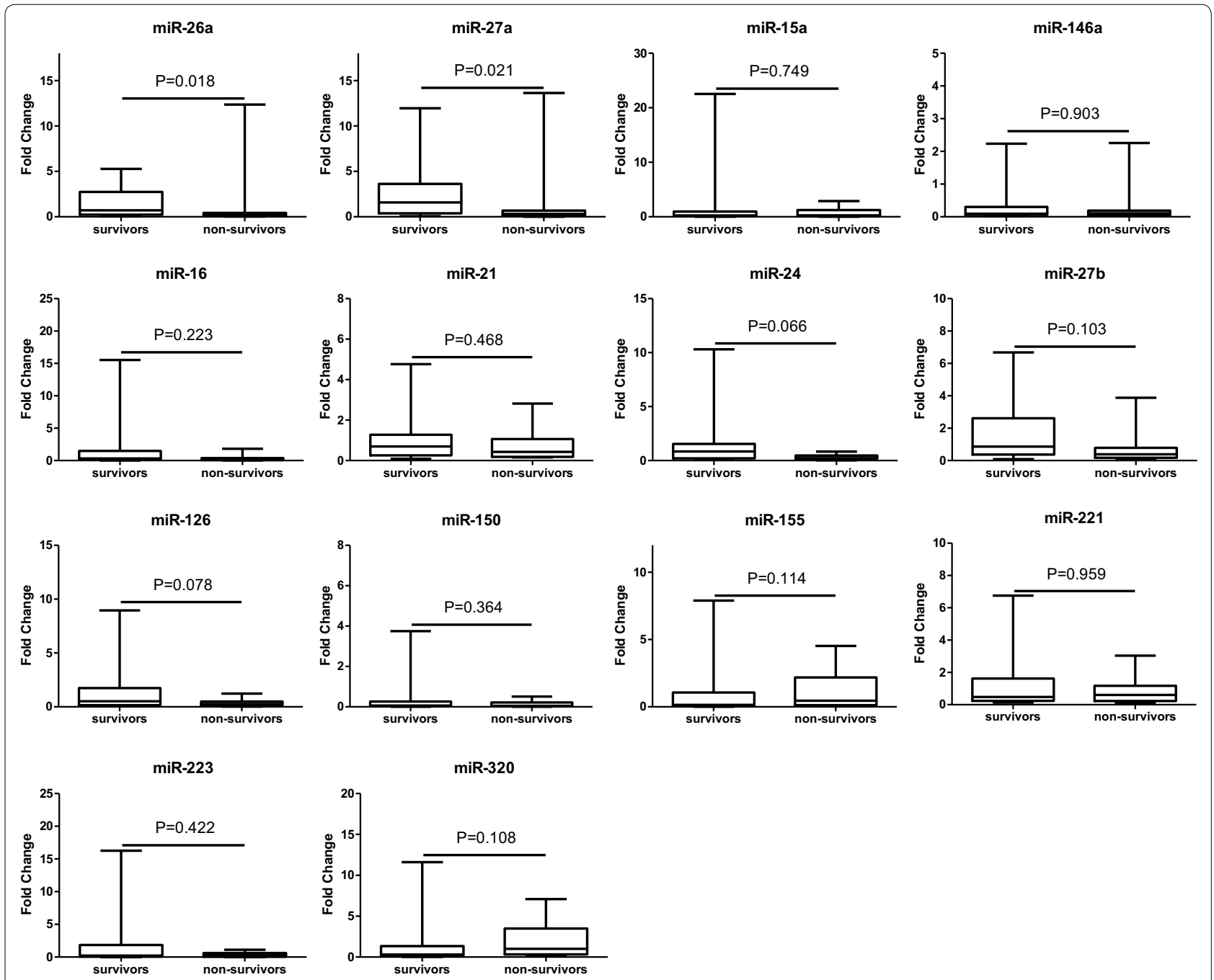

Fig. 4 Comparison of MSC-VEC-miRNAs between survival group and death group in ARDSp patients. Data presented as a relative fold change between ARDSp and ARDSexp for each miRNA. Box plots are displayed where the horizontal bar represents the median, the box represents the IQR and the whiskers represent the maximum and minimum values. Comparisons made by Mann-Whitney $U$ test. miRNA microRNA, IQR interquartile range 
vWF

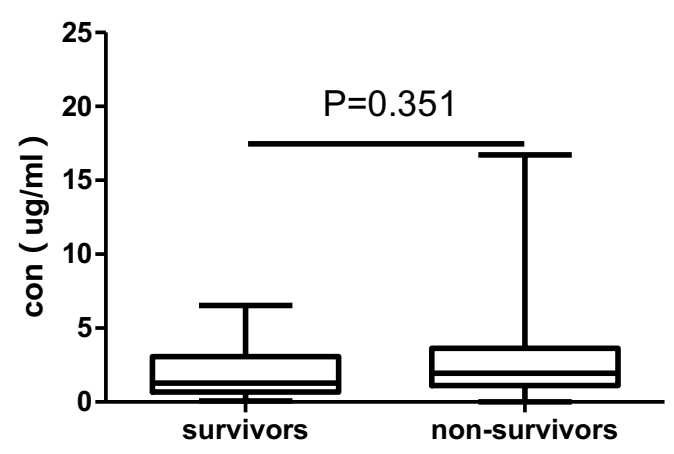

TNF-a

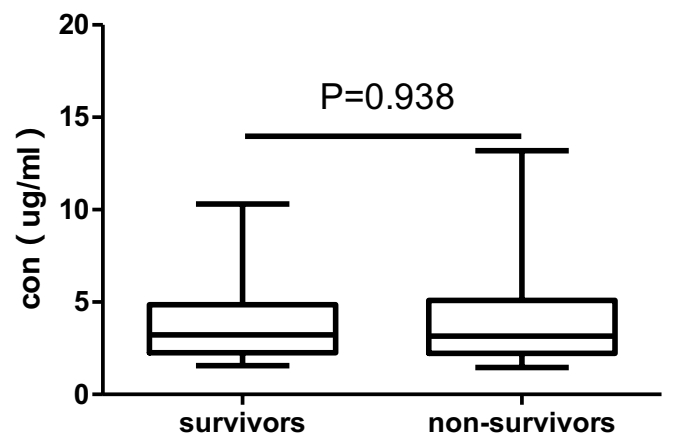

VCAM-1

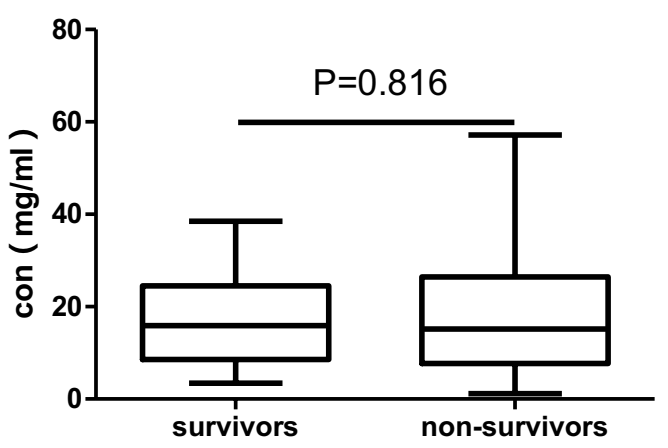

IL-10

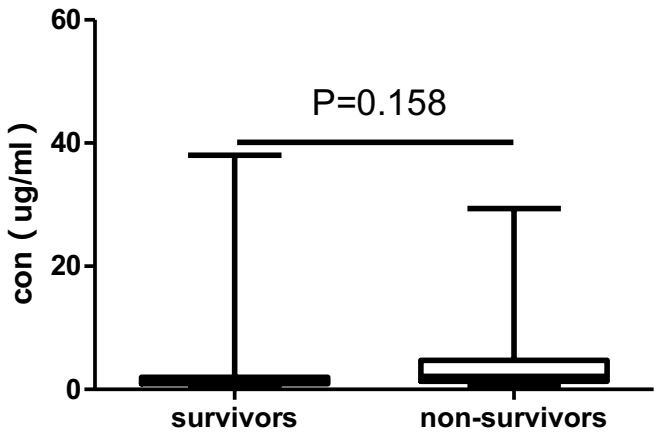

Fig. 5 Comparison of plasma VWF, VCAM-1, IL10, TNFa concentration between survival group and death group in ARDSp patients. The concentration unit of VWF, IL 10, TNFa is ug/ml. The concentration unit of VCAM-1 is mg/ml. Box plots are displayed where the horizontal bar represents the median, the box represents the IQR and the whiskers represent the maximum and minimum values. Comparisons made by Mann-Whitney $U$ test

in ARDSexp group exhibited significantly lower than that in ARDSp group which is inconsistent with expected results. We reviewed forepassed clinical researches and acquired contradictory results with each other. Significant increase in miR-27b expression was observed in the serum samples of patients with peripheral artery disease and arteriosclerosis obliterans when compared to the controls [27, 28]. Coskunpinar et al. [29] reported an increased plasma expression level of miR-221 in acute myocardial infarction compared with healthy controls. However, Tsai et al. presented that stroke patients and atherosclerosis subjects had significantly lower miR-221 serum levels than healthy controls [30]. These conclusions give us a hint that the expression of miRNAs is complex in different diseases originating from the similar pathological change.

Meanwhile, this research explored endothelial markers vWF, VCAM-1 and inflammatory cytokines IL10, TNFa. Plasma vWF concentration in ARDSexp group exhibited significantly lower than that in ARDSp group; however, plasma VCAM-1, IL10, TNF $\alpha$ concentration showed no statistical difference between two groups. As far as we know, endothelium can release vWF which forms additional links between the platelets' glycoprotein and the collagen fibrils. To a certain extent, elevated vWF concentration reflected vascular endothelium lesion. But there was much controversy as to whether vWF could serve as a biomarker for ARDS. VWF is considered as in vivo and in vitro marker of endothelial injury in patients with ARDS [31]. It has previously been reported that high plasma level of vWF was associated with a greater risk of developing ARDS in sepsis patients and was associated with higher mortality in patients with established ARDS [31-34]. It also was reported that plasma levels of vWF did not appear to serve as useful markers for predicting ARDS in patients at risk and mortality in ARDS patients [35-37]. The vWF studies in ARDSp and ARDSexp are rare. Calfee et al. [38] reported that plasma vWF levels were significantly lower in ARDSp than that in ARDSexp which was not consistent with our result. It may be because patients in ARDSexp group were severer with higher APACHE III score and mortality in this study which was not consistent with our research, too. Upregulation of VCAM-1 in endothelial cells by cytokines partly occurs as a result of increased gene TNF $\alpha$ transcription. So, in our results, 

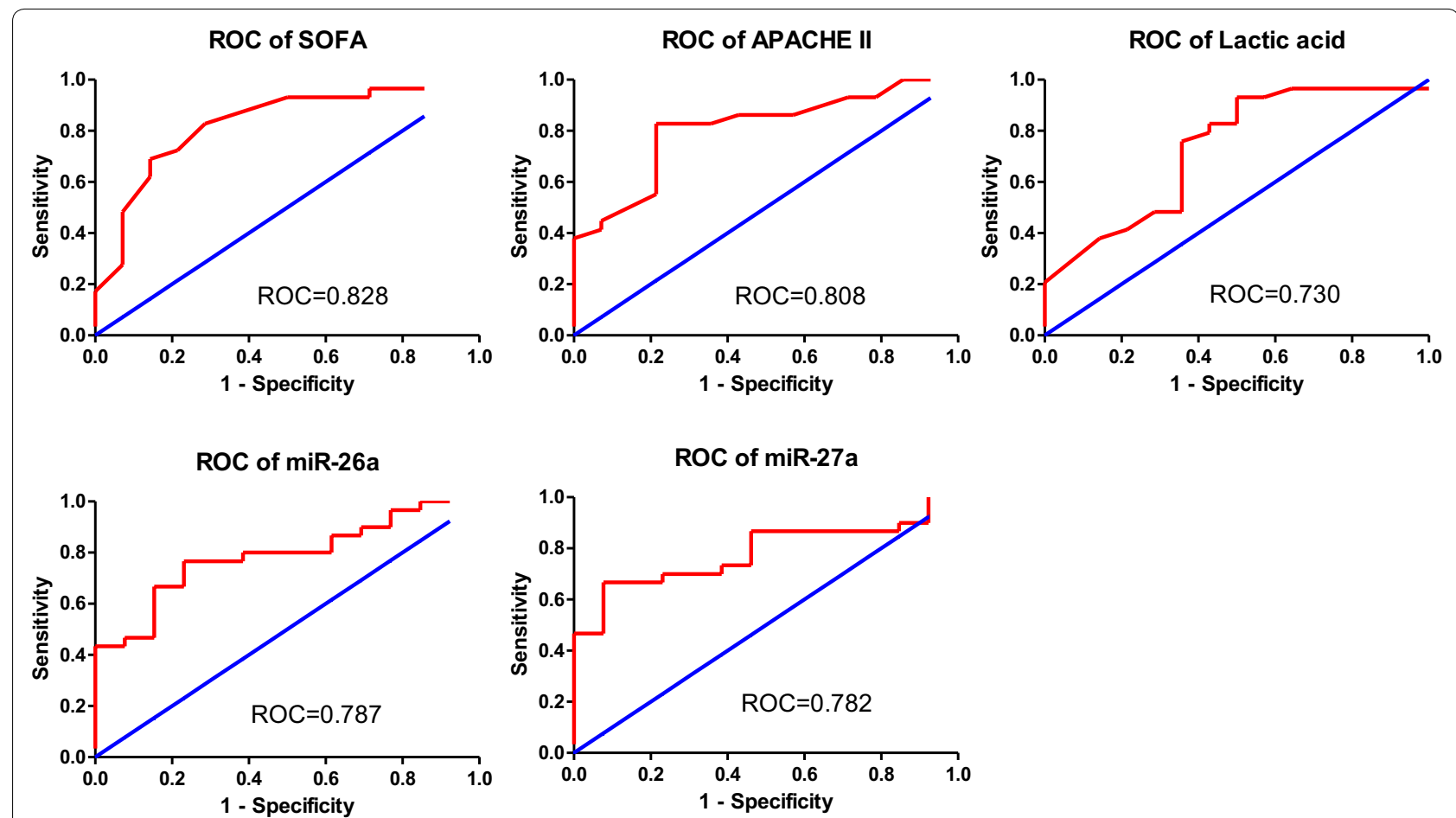

Fig. 6 Receiver operating characteristic curve of SOFA score, APACHE II score, lactic acid value, miR-26a, miR-27a for predicting 28 days mortality in ARDSp patients
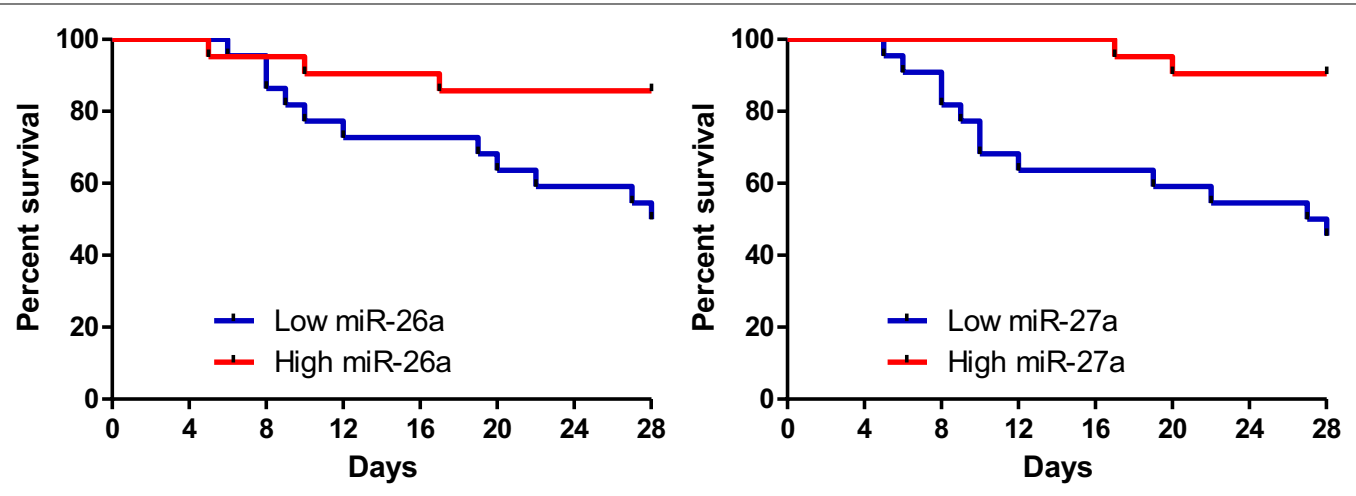

Fig. 7 Probability of survival and subgroup analyses of the risk of death at 28 days

Table 5 Multivariable analysis (miR-26a included) of the death of ARDSp patients

\begin{tabular}{lrll}
\hline & Odds ratio & $\mathbf{9 5 \%} \mathbf{C l}$ & $\boldsymbol{P}$ value \\
\hline (Intercept) & 3931.707 & - & 0.004 \\
Age & 0.959 & $0.908-1.013$ & 0.133 \\
APACHE II score & 0.791 & $0.679-0.921$ & 0.003 \\
miR-26a & 1.483 & $0.999-2.200$ & 0.050 \\
\hline
\end{tabular}

VCAM-1 and TNF $\alpha$ change in the same direction. In our study, leukocyte count, PCT, CRP showed no statistical difference between two groups, which is consistent with the change direction of IL10, TNF $\alpha$.

Significant positive correlation between miR-221 and vWF in plasma levels was found in our study. Circulating is mostly released constitutively from endothelial storage organelles, Weibel-Palade bodies (WPBs) [39, 40]. WPBs are released from endothelial cells in response to a large 
Table 6 Multivariable analysis (miR-27a included) of the death of ARDSp patients

\begin{tabular}{lcll}
\hline & Odds ratio & $\mathbf{9 5 \%} \mathbf{C l}$ & $\boldsymbol{P}$ value \\
\hline (Intercept) & 1673.348 & - & 0.007 \\
Age & 0.964 & $0.914-1.017$ & 0.185 \\
APACHE II score & 0.803 & $0.679-0.926$ & 0.002 \\
miR-27a & 1.425 & $1.008-2.015$ & 0.045 \\
\hline
\end{tabular}

number of agonists which include two distinct groups: those that act by elevating intracellular calcium ion $\left(\mathrm{Ca}^{2+}\right)$ levels and those that act by raising cAMP levels in the cell [41-44]. Xiang et al. [45] identified that miR-24 and miR335 targeted human vWF 3'UTR. Previous studies with regard to miR-221 regulating vWF production are absent. However, miR-221 increases free $\mathrm{Ca}^{2+}$ level of mast cells by PI3 K/Akt/PLC $\gamma / \mathrm{Ca}^{2+}$ signaling pathway [46]. MiR-221 may have the same regulatory role in vascular endothelial cells. The reasons of the positive correlation between miR221 and vWF in plasma levels need to be studied further.

Because the etiology of extrapulmonary ARDS is diverse, we just analyzed plasma $M S C$ - $V E C$-miRNAs and vWF, VCAM-1, IL10, TNF $\alpha$ levels between 28 days survival and 28 days non-survival group in ARDSp patients in order to reduce the heterogeneity between patients. In ARDSp patients, plasma miR-26a and miR-27a levels in non-survival group exhibited significant statistical differences. Plasma levels of miR-26a and miR-27a were lower in non-survival group, which might be because the two miRNAs were protective factors of vascular endothelial cell. APACHE II score, SOFA score, and lactic acid value showed significant statistical differences between two groups. Receiver operating characteristic curve (ROC curve) showed that SOFA score, APACHE II score, lactic acid value, miR-26a, miR-27a roughly equally predict the prognosis of ARDSp patients. Survival curve intuitively points out that plasma miR-26a and miR-27a levels were associated with mortality in ARDSp patients. So, miR-26a and miR-27a may be potential biomarkers for predicting the prognosis of ARDSp patients, the molecular mechanisms behind this which need to be further studied.

There are limitations in this study. Firstly, as stated above, our candidate miRNAs limited to the MSC-VECmiRNAs, which is associated with MSC, has regulatory effects on VEC and has previously been studied in ARDS. The broader miRNA spectrum needs to be involved in future research. Secondly, this study is a clinical observational study, but not involved the molecular mechanism of miRNA regulation in cell. So, we cannot determine where the differential expression of plasma miR-221 and miR-27b come from and which results the difference contributes to. Thirdly, the sample size is relatively small which may have limited the power of statistical difference in this study.

In conclusion, ARDSp patients have higher Murray lung injury score and worse oxygenation index than ARDSexp patients in our study. Plasma miR-221, miR-27b and vWF levels in ARDSexp patients exhibited significantly lower than that in ARDSp patients. Significant positive correlation was found between miR-221 and vWF in plasma levels. In addition, we found plasma miR-26a and miR-27a levels in non-survival group exhibited significantly lower than that in survival group in ARDSp patients.

\section{Additional files}

Additional file 1: Table S1. Summary of candidate miRNAs searched by articles.

Additional file 2: Table S2. Relevant references for candidate miRNAs.

\begin{abstract}
Abbreviations
ARDS: acute respiratory distress syndrome; MSC: mesenchymal stem cells; VEC: vascular endothelial cells; ARDSp: pulmonary ARDS; ARDSexp: extrapulmonary ARDS; miRNAs: microRNAs, miR microRNA; ICU: intensive care unit; VWF: von Willebrand factor; APACHE: acute physiology and chronic health evaluation; SOFA: sequential organ failure assessment; KDIGO: kidney disease improving global outcomes; ECMO: extracorporeal membrane oxygenation; CRRT: continuous renal replacement therapy; VCAM-1: vascular cell adhesion molecule 1; IL: interleukin; TNFa: tumor necrosis factor a; CRP: c-Reactive protein; PCT: procalcitonin; ROC: receiver operating characteristic; AUC: area under the curve; AKI: acute kidney injury; IQR: interquartile range.
\end{abstract}

\section{Authors' contributions}

YZH and $Y Z$ designed the study. YZ, QS, QL, JFX, and CP conducted experimental operation, performed statistical analyses and interpretation of the data. YZ, SQL collect clinical data and wrote the manuscript. LL, YZH commented on the manuscript. All authors read and approved the final manuscript

\section{Author details}

${ }^{1}$ Department of Critical Care Medicine, Zhongda Hospital, School of Medicine, Southeast University, No. 87, Dingjiaqiao Road, Gulou District, Nanjing 210009, China. ${ }^{2}$ Department of Critical Care Medicine, The First Affiliated Hospital of Medical School of Zhejiang University, 79 Qingchun Road, Shangcheng District, Hangzhou 310003, China.

\section{Acknowledgements}

The authors thank Hai-bo Qiu, Yi Yang, Ming Xue, Sen Lu, and Rong-rong Huang for their support in the realization of this study.

\section{Competing interests}

The authors declare that they have no competing interests

\section{Availability of data and materials}

The datasets during and/or analyzed during the current study available from the corresponding author on reasonable request.

Ethics, consent and permissions

This study conformed to the medical ethics standard and was approved by the ethics committee of Zhongda Hospital Affiliated to Southeast University. The document no is 2016ZDSYLL034.0.

\section{Funding}

The study was sponsored by Natural Science Foundation of Jiangsu Province (CN) (BK20141344). 


\section{Publisher's Note}

Springer Nature remains neutral with regard to jurisdictional claims in published maps and institutional affiliations.

Received: 13 November 2017 Accepted: 5 February 2018 Published online: 13 February 2018

\section{References}

1. Bellani G, Laffey JG, Pham T, Fan E, Brochard L, Esteban A, Gattinoni L, van Haren F, Larsson A, McAuley DF, et al. Epidemiology, patterns of care, and mortality for patients with acute respiratory distress syndrome in intensive care units in 50 countries. JAMA. 2016;315(8):788-800.

2. Orfanos SE, Mavrommati I, Korovesi I, Roussos C. Pulmonary endothelium in acute lung injury: from basic science to the critically ill. Intensive Care Med. 2004;30(9):1702-14.

3. Gill SE, Rohan M, Mehta S. Role of pulmonary microvascular endothelial cell apoptosis in murine sepsis-induced lung injury in vivo. Respir Res. 2015;16:109.

4. Gattinoni L, Pelosi P, Suter PM, Pedoto A, Vercesi P, Lissoni A. Acute respiratory distress syndrome caused by pulmonary and extrapulmonary disease. Different syndromes? Am J Respir Crit Care Med. 1998;158(1):3-11.

5. Bernard GR, Artigas A, Brigham KL, Carlet J, Falke K, Hudson L, Lamy M, Legall JR, Morris A, Spragg R. The American-European consensus conference on ARDS. Definitions, mechanisms, relevant outcomes, and clinical trial coordination. Am J Respir Crit Care Med. 1994;149(3 Pt 1):818-24.

6. Rocco PR, Pelosi P. Pulmonary and extrapulmonary acute respiratory distress syndrome: myth or reality? Curr Opin Crit Care. 2008;14(1):50-5.

7. Hoeper MM, Spiekerkoetter E, Westerkamp V, Gatzke R, Fabel H. Intravenous iloprost for treatment failure of aerosolised iloprost in pulmonary arterial hypertension. Eur Respir J. 2002;20(2):339-43.

8. Goodman LR, Fumagalli R, Tagliabue P, Tagliabue M, Ferrario M, Gattinoni $L$, Pesenti A. Adult respiratory distress syndrome due to pulmonary and extrapulmonary causes: CT, clinical, and functional correlations. Radiology. 1999;213(2):545-52.

9. Rialp G, Betbese AJ, Perez-Marquez M, Mancebo J. Short-term effects of inhaled nitric oxide and prone position in pulmonary and extrapulmonary acute respiratory distress syndrome. Am J Respir Crit Care Med. 2001;164(2):243-9.

10. Lim CM, Kim EK, Lee JS, Shim TS, Lee SD, Koh Y, Kim WS, Kim DS, Kim WD Comparison of the response to the prone position between pulmonary and extrapulmonary acute respiratory distress syndrome. Intensive Care Med. 2001;27(3):477-85.

11. Riva DR, Oliveira MB, Rzezinski AF, Rangel G, Capelozzi VL, Zin WA, Morales MM, Pelosi P, Rocco PR. Recruitment maneuver in pulmonary and extrapulmonary experimental acute lung injury. Crit Care Med. 2008;36(6):1900-8.

12. Kloot TE, Blanch L, Melynne Youngblood A, Weinert C, Adams AB, Marin $\mathrm{JJ}$, Shapiro RS, Nahum A. Recruitment maneuvers in three experimental models of acute lung injury. Effect on lung volume and gas exchange. Am J Respir Crit Care Med. 2000;161(5):1485-94.

13. Tugrul S, Akinci O, Ozcan PE, Ince S, Esen F, Telci L, Akpir K, Cakar N. Effects of sustained inflation and postinflation positive end-expiratory pressure in acute respiratory distress syndrome: focusing on pulmonary and extrapulmonary forms. Crit Care Med. 2003;31(3):738-44.

14. Pati S, Gerber MH, Menge TD, Wataha KA, Zhao Y, Baumgartner JA, Zhao J, Letourneau PA, Huby MP, Baer LA, et al. Bone marrow derived mesenchymal stem cells inhibit inflammation and preserve vascular endothelial integrity in the lungs after hemorrhagic shock. PLOS ONE. 2011;6(9):e25171.

15. Yang Y, Chen QH, Liu AR, Xu XP, Han JB, Qiu HB. Synergism of MSCsecreted HGF and VEGF in stabilising endothelial barrier function upon lipopolysaccharide stimulation via the Rac1 pathway. Stem Cell Res Ther. 2015;6:250.

16. Hu S, Li J, Xu X, Liu A, He H, Xu J, Chen Q, Liu S, Liu L, Qiu H, et al. The hepatocyte growth factor-expressing character is required for mesenchymal stem cells to protect the lung injured by lipopolysaccharide in vivo. Stem Cell Res Ther. 2016;7(1):66

17. Wang H, Zheng R, Chen Q, Shao J, Yu J, Hu S. Mesenchymal stem cells microvesicles stabilize endothelial barrier function partly mediated by hepatocyte growth factor (HGF). Stem Cell Res Ther. 2017;8(1):211.
18. Potter DR, Miyazawa BY, Gibb SL, Deng X, Togaratti PP, Croze RH, Srivastava AK, Trivedi A, Matthay M, Holcomb JB, et al. Mesenchymal stem cell derived extracellular vesicles attenuate pulmonary vascular permeability and lung injury induced by hemorrhagic shock and trauma. J Trauma Acute Care Surg. 2017:84(2):245-56.

19. Liu L, He H, Liu A, Xu J, Han J, Chen Q, Hu S, Xu X, Huang Y, Guo F, et al. Therapeutic effects of bone marrow-derived mesenchymal stem cells in models of pulmonary and extrapulmonary acute lung injury. Cell Transplant. 2015;24(12):2629-42.

20. Araujo IM, Abreu SC, Maron-Gutierrez T, Cruz F, Fujisaki L, Carreira H Jr, Ornellas F, Ornellas D, Vieira-de-Abreu A, Castro-Faria-Neto HC, et al. Bone marrow-derived mononuclear cell therapy in experimental pulmonary and extrapulmonary acute lung injury. Crit Care Med. 2010;38(8):1733-41.

21. Zhou Z, You Z. Mesenchymal stem cells alleviate LPS-induced acute lung injury in mice by MiR-142a-5p-controlled pulmonary endothelial cell autophagy. Cell Physiol Biochem. 2016;38(1):258-66.

22. Bao H, Gao F, Xie G, Liu Z. Angiotensin-converting enzyme 2 inhibits apoptosis of pulmonary endothelial cells during acute lung injury through suppressing MiR-4262. Cell Physiol Biochem. 2015;37(2):759-67.

23. Force ADT, Ranieri VM, Rubenfeld GD, Thompson BT, Ferguson ND, Caldwell E, Fan E, Camporota L, Slutsky AS. Acute respiratory distress syndrome: the Berlin definition. JAMA. 2012;307(23):2526-33.

24. Kim SJ, Oh BJ, Lee JS, Lim CM, Shim TS, Lee SD, Kim WS, Kim DS, Kim WD, Koh Y. Recovery from lung injury in survivors of acute respiratory distress syndrome: difference between pulmonary and extrapulmonary subtypes. Intensive Care Med. 2004;30(10):1960-3.

25. Morisawa K, Fujitani S, Taira Y, Kushimoto S, Kitazawa Y, Okuchi K, Ishikura H, Sakamoto T, Tagami T, Yamaguchi J, et al. Difference in pulmonary permeability between indirect and direct acute respiratory distress syndrome assessed by the transpulmonary thermodilution technique: a prospective, observational, multi-institutional study. J Intensive Care. 2014;2(1):24.

26. Luo L, Shaver CM, Zhao Z, Koyama T, Calfee CS, Bastarache JA, Ware LB. Clinical predictors of hospital mortality differ between direct and indirect ARDS. Chest. 2017;151(4):755-63.

27. Signorelli SS, Volsi GL, Pitruzzella A, Fiore V, Mangiafico M, Vanella L, Parenti R, Rizzo M, Volti GL. Circulating miR-130a, miR-27b, and miR-210 in patients with peripheral artery disease and their potential relationship with oxidative stress. Angiology. 2016;67(10):945-50.

28. Li T, Cao H, Zhuang J, Wan J, Guan M, Yu B, Li X, Zhang W. Identification of miR-130a, miR-27b and miR-210 as serum biomarkers for atherosclerosis obliterans. Clin Chim Acta. 2011;412(1-2):66-70.

29. Coskunpinar E, Cakmak HA, Kalkan AK, Tiryakioglu NO, Erturk M, Ongen Z. Circulating miR-221-3p as a novel marker for early prediction of acute myocardial infarction. Gene. 2016;591(1):90-6.

30. Tsai PC, Liao YC, Wang YS, Lin HF, Lin RT, Juo SH. Serum microRNA-21 and microRNA-221 as potential biomarkers for cerebrovascular disease. J Vasc Res. 2013;50(4):346-54

31. Ware LB, Eisner MD, Thompson BT, Parsons PE, Matthay MA. Significance of von Willebrand factor in septic and nonseptic patients with acute lung injury. Am J Respir Crit Care Med. 2004;170(7):766-72.

32. Rubin DB, Wiener-Kronish JP, Murray JF, Green DR, Turner J, Luce JM, Montgomery AB, Marks JD, Matthay MA. Elevated von Willebrand factor antigen is an early plasma predictor of acute lung injury in nonpulmonary sepsis syndrome. J Clin Invest. 1990;86(2):474-80.

33. Ware LB, Conner ER, Matthay MA. von Willebrand factor antigen is an independent marker of poor outcome in patients with early acute lung injury. Crit Care Med. 2001;29(12):2325-31.

34. Siemiatkowski A, Kloczko J, Galar M, Czaban S. von Willebrand factor antigen as a prognostic marker in posttraumatic acute lung injury. Haemostasis. 2000:30(4):189-95.

35. Bajaj MS, Tricomi SM. Plasma levels of the three endothelial-specific proteins von Willebrand factor, tissue factor pathway inhibitor, and thrombomodulin do not predict the development of acute respiratory distress syndrome. Intensive Care Med. 1999;25(11):1259-66.

36. Moss M, Ackerson L, Gillespie MK, Moore FA, Moore EE, Parsons PE. von Willebrand factor antigen levels are not predictive for the adult respiratory distress syndrome. Am J Respir Crit Care Med. 1995;151(1):15-20.

37. Cartin-Ceba R, Hubmayr RD, Qin R, Peters S, Determann RM, Schultz MJ, Gajic O. Predictive value of plasma biomarkers for mortality and organ failure development in patients with acute respiratory distress syndrome. J Crit Care. 2015;30(1):219.e1-7. 
38. Calfee CS, Janz DR, Bernard GR, May AK, Kangelaris KN, Matthay MA, Ware LB. Distinct molecular phenotypes of direct vs indirect ARDS in singlecenter and multicenter studies. Chest. 2015;147(6):1539-48.

39. Valentijn KM, Eikenboom J. Weibel-Palade bodies: a window to von Willebrand disease. J Thromb Haemost. 2013;11(4):581-92.

40. van den Biggelaar M, Hernandez-Fernaud JR, van den Eshof BL, Neilson LJ, Meijer AB, Mertens K, Zanivan S. Quantitative phosphoproteomics unveils temporal dynamics of thrombin signaling in human endothelial cells. Blood. 2014:123(12):e22-36.

41. Vischer UM, Barth H, Wollheim CB. Regulated von Willebrand factor secretion is associated with agonist-specific patterns of cytoskeletal remodeling in cultured endothelial cells. Arterioscler Thromb Vasc Biol. 2000;20(3):883-91.

42. van den Eijnden-Schrauwen Y, Atsma DE, Lupu F, de Vries RE, Kooistra T, Emeis JJ. Involvement of calcium and G proteins in the acute release of tissue-type plasminogen activator and von Willebrand factor from cultured human endothelial cells. Arterioscler Thromb Vasc Biol. 1997;17(10):2177-87.
43. Vischer UM, Wollheim CB. Epinephrine induces von Willebrand factor release from cultured endothelial cells: involvement of cyclic AMPdependent signalling in exocytosis. Thromb Haemost. 1997;77(6):1182-8.

44. Kaufmann JE, Oksche A, Wollheim CB, Gunther G, Rosenthal W, Vischer UM. Vasopressin-induced von Willebrand factor secretion from endothelial cells involves V2 receptors and CAMP. J Clin Invest. 2000;106(1):107-16.

45. Xiang Y, Cheng J, Wang D, Hu X, Xie Y, Stitham J, Atteya G, Du J, Tang WH, Lee $\mathrm{SH}$, et al. Hyperglycemia repression of miR-24 coordinately upregulates endothelial cell expression and secretion of von Willebrand factor. Blood. 2015;125(22):3377-87.

46. Xu H, Gu LN, Yang QY, Zhao DY, Liu F. MiR-221 promotes IgE-mediated activation of mast cells degranulation by PI3 K/Akt/PLCgamma/Ca(2+) pathway. J Bioenerg Biomembr. 2016;48(3):293-9.

\section{Submit your manuscript to a SpringerOpen ${ }^{\circ}$ journal and benefit from:}

- Convenient online submission

- Rigorous peer review

- Open access: articles freely available online

- High visibility within the field

Retaining the copyright to your article

Submit your next manuscript at $\boldsymbol{\nabla}$ springeropen.com 